\title{
7 EFICÁCIA DOS PROGRAMAS PSICOEDUCACIONAIS NA SOBRECARGA NOS FAMILIARES CUIDADORES DE PESSOAS COM DEMÊNCIA: REVISÃO INTEGRATIVA
}

\author{
| Mafalda Silva ${ }^{1}$; Luís Sá2 ${ }^{2}$ Lia Sousa ${ }^{3} \mid$
}

\section{RESUMO}

CONTEXTO: As demências causam grande impacto no seio familiar, aumentando a sobrecarga emocional, de todos os membros do agregado familiar, mas principalmente do cuidador e gerando conflitos na dinâmica familiar. Como tal, torna-se emergente a necessidade de implementação de programas de apoio para os cuidadores familiares.

OBJETIVO: Avaliar qual a eficácia dos programas psicoeducacionais na sobrecarga dos cuidadores de pessoas com demência.

MÉTODOS: Revisão integrativa da literatura sobre programas psicoeducacionais para cuidadores, publicados entre janeiro de 2010 e dezembro de 2015 nas bases de dados PubMed e b-on, utilizando as seguintes palavras-chave: "psychoeducation, dementia, caregiver, burden", "psicoeducação, cuidador, demência, sobrecarga".

RESULTADOS: Foram analisados 9 artigos que avaliam os efeitos da aplicação de um programa psicoeducacional na sobrecarga dos familiares de cuidadores de pacientes com demência. Verifica-se que existe uma melhoria substancial do bem-estar dos cuidadores; um aumento da aquisição de estratégias de resolução de problemas e de técnicas de gestão de stress; aumento do conhecimento acerca dos recursos na comunidade e de como lidar com situações de crise nas diferentes fases da doença.

CONCLUSÃO: Os resultados sugerem que a aplicação de programas psicoeducacionais em cuidadores familiares de pessoas com demência tem resultados positivos na sobrecarga do cuidador e na melhoria do seu bem-estar.

PALAVRAS-CHAVE: Cuidadores familiares; Demência; Sobrecarga; Psicoeducação

\section{RESUMEN}

"Efectividad de los programas psicoeducativos en la carga de los cuidadores de personas con demencia de la familia: Una revisión integradora"

CONTEXTO: Las demencias causan gran impacto en el seno familiar, aumentando la sobrecarga emocional, de todos los miembros del hogar, pero principalmente del cuidador y generando conflictos en la dinámica familiar. Como tal, se hace emergente la necesidad de implementar programas de apoyo para los cuidadores familiares. Se entiende por sobrecarga del cuidador un conjunto de problemas físicos, psicológicos, emocionales, sociales y financieros experimentados por cuidadores de pacientes con algún tipo de compromiso.

OBJETIVO: Evaluar cuál es la eficacia de los programas psicoeducativos en la sobrecarga de los cuidadores de personas con demencia.

MÉTODOS: Revisión integrada de artículos sobre la literatura programas psicoeducativo para cuidadores, publicados entre enero de 2010 y diciembre de 2015, PubMed y b-On, utilizando las siguientes palabras: "psicoeducación, la demencia, el cuidador, carga" "psicoeducación, cuidador, la demencia, la sobrecarga".

RESULTADOS: Se analizaron 9 artículos que evalúan los efectos de la aplicación de un programa psicoeducativo en la sobrecarga de los familiares de cuidadores de pacientes con demencia. Parece que hay una mejora sustancial del bienestar de los cuidadores; un aumento en la adquisición de estrategias de resolución de problemas y técnicas de manejo del estrés; un mayor conocimiento acerca de los recursos de la comunidad y cómo hacer frente a situaciones de crisis en las diferentes etapas de la enfermedad. CONCLUSIÓN: Los resultados sugieren que la aplicación de programas psicoeducativos en cuidadores familiares de personas con demencia tiene resultados positivos en la sobrecarga del cuidador y en la mejora de su bienestar.

\section{DESCRIPTORES: Cuidadores familiares; Demencia; Sobre- carga; Psicoeducación}

\begin{abstract}
"Efficacy of psychoeducational programs in the overload of family caregivers of people with dementia: Integrative review"

BACKGROUND: Dementias have a major impact on the family, increasing the emotional overload of all household members, but mainly the caregiver and generating conflicts in the family dynamics. As such, the need to implement support programs for family caregivers emerges. Caregiver overload is understood as a set of physical, psychological, emotional, social and financial problems experienced by caregivers of patients with some type of impairment.

AIM: To evaluate the effectiveness of psychoeducational programs in the overload of caregivers of people with dementia.

METHODS: Integrative review of the literature on psychoeducational programs for caregivers, published between January 2010 and December 2015 in the PubMed and b-on databases, using the following keywords: "psychoeducation, dementia, caregiver, burden".

RESULTS: We analyzed 9 articles that evaluate the effects of the application of a psychoeducational program on the overload of family members of caregivers of patients with dementia. There is a substantial improvement in the well-being of caregivers; An increase in the acquisition of problemsolving strategies and stress-management techniques; Increase knowledge about resources in the community and how to deal with crisis situations in the different phases of the disease.

CONCLUSION: The results suggest that the application of psychoeducational programs in family caregivers of people with dementia has positive results in the caregiver's overload and in the improvement of their wellbeing.
\end{abstract}

\section{KEYWORDS: Family caregivers; Dementia; Burden; Psycho- education}

Submetido em 30-09-2017

Aceite em 22-01-2018

1 Doutoranda na Universidade Católica Portuguesa, Instituto de Ciências da Saúde - Porto, Centro de Investigação Interdisciplinar em Saúde, Assistente na Escola Superior de Saúde de Santa Maria, mafaldassilva@hotmail.com

2 Professor Auxiliar na Universidade Católica Portuguesa, Instituto de Ciências da Saúde - Porto, Centro de Investigação Interdisciplinar em Saúde, lsa@porto.ucp.pt 3 Doutoranda em Ciências de Enfermagem na Universidade do Porto, ICBAS; Enfermeira especialista e Mestre em Enfermagem de Saúde Mental e Psiquiatria; Assistente Convidada na Escola Superior de Enfermagem do Porto; Investigadora no CINTESIS; Enfermeira no Centro Hospitalar de São João, liasousa_27@hotmail.com

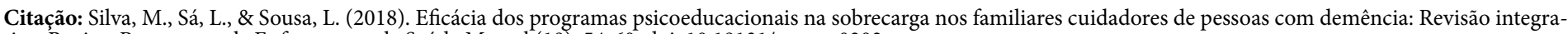
tiva. Revista Portuguesa de Enfermagem de Saúde Mental (19), 54-60. doi: 10.19131/rpesm.0202 


\section{INTRODUÇÃO}

A incidência da demência a nível global tem aumentado exponencialmente ao longo das últimas décadas. A nível mundial, prevê-se que em 2050 existam 115,4 milhões de pessoas com demência (HelpAge International, 2017).

A demência (transtorno neurocognitivo major) é uma síndrome, que implica deterioração da cognição, alterações do comportamento e prejuízo funcional (American Psychiatric Association, 2013). Apesar do caracter progressivo da demência é necessário continuar a estimular o envolvimento das pessoas afetadas nas atividades de vida diária, de forma a manter as suas capacidades o maior tempo possível.

Atualmente, a maioria das pessoas com demência são cuidadas pelos familiares, o que acarreta sobrecarga física, emocional, social e económica (American Psychiatric Association, 2013), por isso é urgente implementar programas de apoio para os cuidadores familiares de pessoas com demência a residir no domicílio.

A sobrecarga do cuidador entende-se como um conjunto de problemas físicos, psicológicos, emocionais, sociais e financeiros, experienciados por cuidadores de pacientes com algum tipo de comprometimento comprometendo a capacidade para lidar com o paciente. A saúde mental dos cuidadores familiares tende a ser afetada, aumentam os sentimentos de desesperança, quadros depressivos e irritabilidade emocional que advém da necessidade de prestarem cuidados diariamente (Marques, 2007).

Atualmente, a psicoeducação tem assumido especial destaque na intervenção com cuidadores familiares. As intervenções psicoeducativas visam dotar os cuidadores de conhecimentos e capacidades para cuidar e a sua implementação tem vindo a demostrando uma diminuição da sobrecarga emocional, física, um aumento substancial do conhecimento da doença e de estratégias para lidar com a sintomatologia da pessoa com demência (Cardoso, 2011). A psicoeducação integra uma componente educativa e de suporte, numa perspetiva multidisciplinar caracterizando-se por ser delimitada no tempo, estruturada em sessões, focada na atualidade e na técnica de resolução dos problemas, incide principalmente na literacia sobre a demência, sobre os sinais e sintomas, diagnóstico, etiologia, prognóstico, tratamentos farmacológicos e não farmacológicos, retardamento da institucionalização e apoios sociais (Sousa, Mendes, e Relvas 2007).
O objetivo deste estudo foi avaliar qual a eficácia dos programas de psicoeducação na sobrecarga dos cuidadores de pessoas com demência.

\section{MÉTODOS}

O método utilizado foi a revisão integrativa da literatura, de acordo com os passos sugeridos por Mendes, Silveira e Galvão (2008). Com a revisão procurou-se responder à pergunta "Qual a eficácia dos programas psicoeducacionais na sobrecarga dos cuidadores de pessoas com demência?". Utilizaram-se as seguintes palavras-chave: psychoeducation, family caregivers, burden, dementia e os respetivos termos em Português. Os estudos foram pesquisados nas bases de dados PubMed e b-on. Na base de dados Pubmed foram utilizadas as seguintes combinações: (psychoeducational[All Fields] AND ("family caregivers" [MeSH Terms] OR "family caregivers"[All Fields]) AND burden[All Fields] AND ("dementia"[MeSH Terms] OR "dementia"[All Fields])) AND ("loattrfree full text"[sb] AND “2010/12/08”[PDat] : “2015/12/06"[PDat] AND (English[lang] OR Portuguese[lang]) AND (medline[sb] OR jsubsetn[text])). Os critérios de inclusão foram: artigos publicados entre 2010 e 2015, em língua portuguesa e inglesa, revisões sistemáticas relevantes para o estudo da problemática, artigos originais e randomizados publicados entre 2010 e 2015 e disponíveis em free full text. Os artigos foram lidos na íntegra e analisados segundo os objetivos, tipo de estudo, amostragem e os resultados obtidos. Foram excluídos os artigos relativos a estudos de caso, relato de experiências, estudos de intervenções psicoeducacionais aplicados em outros diagnósticos e, após a leitura na íntegra dos artigos, foram excluídos os artigos que não apresentavam o programa estruturado nem os instrumentos utilizados.

\section{RESULTADOS}

Foram encontrados 6 artigos na base PubMed e 3 artigos na b-on, tendo sido incluídos 9 artigos, de acordo com os critérios de inclusão estabelecidos. Os estudos encontrados $(n=9)$ apresentaram características diferentes quanto aos modelos teóricos e metodológicos utilizados, no entanto, verifica-se a utilização frequente das abordagens teóricas de Lazarus e Folkman (1984). Na Fig. 1 apresentamos a seleção de estudos realizada segundo os critérios de inclusão estabelecidos. 
Figura 1 - Seleção dos estudos publicados sobre intervenções psicoeducacionais em cuidadores de pacientes com demência

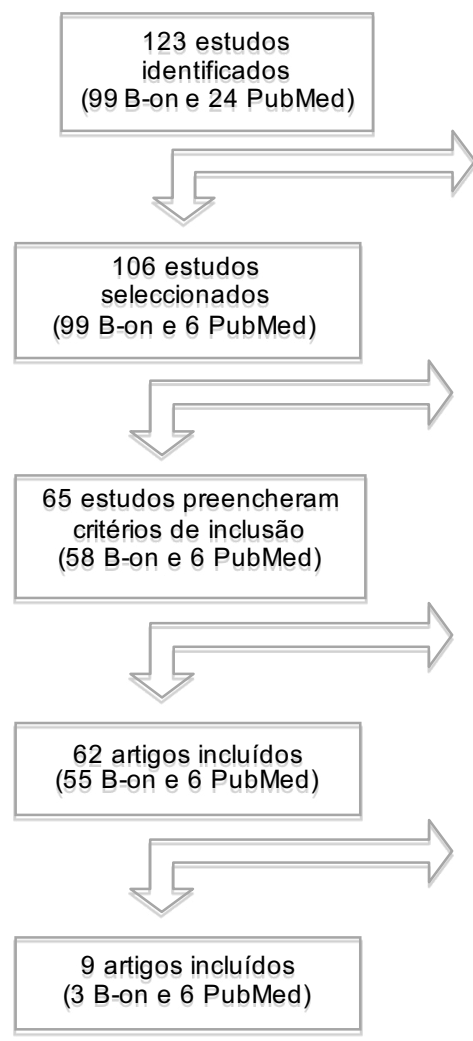

Da pesquisa realizada foram identificados 9 estudos $(\mathrm{n}=3$ em Portugal; $\mathrm{n}=1$ Brasil; $\mathrm{n}=1$ Hong Kong; $\mathrm{n}=1$ Canadá, $\mathrm{n}=1$ Perú, $\mathrm{n}=1$ Reino Unido e por último $\mathrm{n}=1$ proveniente da Alemanha, Suíça e Áustria). Estes apresentam diferentes objetivos, de forma, a estudar a sobrecarga nos cuidadores, embora alguns estudos incluam a díade cuidador-paciente. Relativamente às metodologias utilizadas são estudos quantitativos com a aplicação de diferentes instrumentos de avaliação. Quanto à duração das sessões é variável, de acordo, com os conteúdos programáticos que foram transmitidos. Os artigos identificados encontram-se detalhados no Quadro 1.

Quadro 1 -Artigos Incluídos na Revisão

\begin{tabular}{|c|c|c|c|c|c|c|c|}
\hline $\begin{array}{l}\text { Autor(es) } \\
\text { (Ano) }\end{array}$ & Origem & Objetivos & $\begin{array}{l}\text { População / } \\
\text { amostra }\end{array}$ & Método & Instrumentos & $\begin{array}{l}\text { Tipo de } \\
\text { intervenção } \\
\text { / Duração }\end{array}$ & $\begin{array}{l}\text { Resultados: } \\
\text { Demonstra- } \\
\text { ção da eficácia } \\
\text { do programa }\end{array}$ \\
\hline $\begin{array}{l}\text { Alves, } \\
\text { Brandão, } \\
\text { Teixeira, Aze- } \\
\text { vedo, Duarte, } \\
\text { Ribeiro e Paúl } \\
(2015)\end{array}$ & $\begin{array}{l}\text { Portugal } \\
\text { (Cuidar } \\
\text { em Casa) }\end{array}$ & $\begin{array}{l}\text { Avaliar a } \\
\text { saúde física e } \\
\text { mental, sobre- } \\
\text { carga, distress } \\
\text { psicológico e } \\
\text { gratificação; }\end{array}$ & 53 Cuidadores & $\begin{array}{l}\text { Estudo } \\
\text { clínico } \\
\text { random- } \\
\text { izado } \\
\text { controlado }\end{array}$ & $\begin{array}{l}\text { Informação sociodemográfica; Medi- } \\
\text { cal Outcomes Survey -Short-Form } 12 \\
\text { Health Survey; Modified Caregiver } \\
\text { Strain Index; Escala dos Aspetos } \\
\text { Positivos do Cuidar; General Health } \\
\text { Questionnaire. }\end{array}$ & $\begin{array}{l}7 \text { Sessões } \\
\text { semanais de } \\
120 \text { minutos } \\
\text { cada } \\
\text { Total de } 14 \mathrm{~h} .\end{array}$ & $\begin{array}{l}\text { Diminuição do } \\
\text { stress psicológi- } \\
\text { co; }\end{array}$ \\
\hline $\begin{array}{l}\text { Pereira, F. } \\
(2015)\end{array}$ & $\begin{array}{l}\text { Portugal } \\
\text { (CUIDA) }\end{array}$ & $\begin{array}{l}\text { Reduzir os } \\
\text { níveis de } \\
\text { sobrecarga do } \\
\text { cuidador; }\end{array}$ & $\begin{array}{l}\text { Entre } 8 \text { e } 16 \\
\text { díades de } \\
\text { cuidadores e } \\
\text { Paciente }\end{array}$ & $\begin{array}{l}\text { Estudo } \\
\text { clínico } \\
\text { random- } \\
\text { izado } \\
\text { controlado }\end{array}$ & $\begin{array}{l}\text { Cuidador: Informação Biográfica; } \\
\text { Escala de Zarit; Questionário de Neces- } \\
\text { sidades Paciente: Escala de Barthel; } \\
\text { Indice de Lawton e Brody; Mini Mental } \\
\text { Scale; Global Deterioration Scale. }\end{array}$ & $\begin{array}{l}8 \text { Sessões de } \\
180 \text { minutos } \\
\text { semanais. }\end{array}$ & $\begin{array}{l}\text { Sobrecarga } \\
\text { aumenta com } \\
\text { a evolução da } \\
\text { doença. }\end{array}$ \\
\hline $\begin{array}{l}\text { Livingston, } \\
\text { G., Barber, J., } \\
\text { Rapaport, P., } \\
\text { Knapp, M., } \\
\text { Griffin, M., } \\
\text { King, D., \& } \\
\text { Cooper, C } \\
\text { (2013) }\end{array}$ & $\begin{array}{l}\text { Reino } \\
\text { Unido } \\
\text { (Pro- } \\
\text { grama } \\
\text { START) }\end{array}$ & $\begin{array}{l}\text { Avaliar a } \\
\text { ansiedade e } \\
\text { depressão no } \\
\text { cuidador. }\end{array}$ & $\begin{array}{l}260 \text { Cuida- } \\
\text { dores }\end{array}$ & $\begin{array}{l}\text { Estudo } \\
\text { clínico } \\
\text { random- } \\
\text { izado } \\
\text { controlado }\end{array}$ & $\begin{array}{l}\text { Hospital anxiety and depression scale; } \\
\text { Health status questionnaire mental } \\
\text { health domain; Quality of life Alzheim- } \\
\text { er' s disease; Modified conflict tactics } \\
\text { scale. }\end{array}$ & $\begin{array}{l}8 \text { Sessões } \\
\text { semanais/ } 90 \\
\text { minutos. }\end{array}$ & $\begin{array}{l}\text { Melhoria na } \\
\text { qualidade de } \\
\text { vida dos cuida- } \\
\text { dores; }\end{array}$ \\
\hline $\begin{array}{l}\text { Ducharme, } \\
\text { Lachance, } \\
\text { Lévesque, Ker- } \\
\text { goat, \& Zarit } \\
\text { (2012) }\end{array}$ & Canadá & $\begin{array}{l}\text { Estudar } \\
\text { os efeitos } \\
\text { positivos da } \\
\text { aplicação do } \\
\text { programa; }\end{array}$ & 97 Cuidadores & $\begin{array}{l}\text { Estudo } \\
\text { clínico } \\
\text { random- } \\
\text { izado } \\
\text { controlado }\end{array}$ & $\begin{array}{l}\text { 8-item Preparedness for Caregiving } \\
\text { Scale; Self-Efficacy Scale; Revised Scale } \\
\text { for Caregiving Self-Efficacy; Planning } \\
\text { for Future Care Needs Scale; Knowledge } \\
\text { of Services Scale; The Carers' Assess- } \\
\text { ment of Managing Index; } 27 \text {-item } \\
\text { Inventory of Socially Supportive Be- } \\
\text { haviors; The Family Caregiver Conflict } \\
\text { Scale; Psychological Distress Index. }\end{array}$ & $\begin{array}{l}7 \text { Sessões } \\
\text { individuais/ } \\
\text { semanais } \\
\text { com duração } \\
\text { de 90min. }\end{array}$ & $\begin{array}{l}\text { Melhoria pouco } \\
\text { significativa nos } \\
\text { indicadores de } \\
\text { saúde do cuida- } \\
\text { dor e qualidade } \\
\text { de vida; }\end{array}$ \\
\hline
\end{tabular}




\begin{tabular}{|c|c|c|c|c|c|c|c|}
\hline $\begin{array}{l}\text { Lopes e Ca- } \\
\text { chioni (2012) }\end{array}$ & Brasil & $\begin{array}{l}\text { Avaliar o im- } \\
\text { pacto do pro- } \\
\text { grama para } \\
\text { cuidadores de } \\
\text { pessoas com } \\
\text { demência, } \\
\text { relaciona- } \\
\text { ndo com o } \\
\text { bem-estar } \\
\text { subjetivo. }\end{array}$ & 21 Cuidadores & $\begin{array}{l}\text { Estudo } \\
\text { clínico } \\
\text { random- } \\
\text { izado } \\
\text { controlado }\end{array}$ & $\begin{array}{l}\text { Ficha de caracterização sociodemo- } \\
\text { gráfica; } \\
\text { Escala de satisfação geral com a } \\
\text { vida; } \\
\text { Escala de ânimo positivo e negativo; } \\
\text { Escala de depressão geriátrica. }\end{array}$ & $\begin{array}{l}\text { Entrevistas } \\
\text { antes e depois } \\
\text { da aplicação } \\
\text { do programa; } \\
15 \text { Sessões }\end{array}$ & $\begin{array}{l}\text { Impacto positivo } \\
\text { no bem-estar } \\
\text { subjetivo, na } \\
\text { satisfação geral } \\
\text { com a vida, no } \\
\text { envolvim- } \\
\text { ento social e no } \\
\text { equilíbrio entre } \\
\text { afetos positivos e } \\
\text { negativos. }\end{array}$ \\
\hline $\begin{array}{l}\text { Wang, \& } \\
\text { Chien (2011) }\end{array}$ & $\begin{array}{l}\text { Hong Kong } \\
\text { (Family Mu- } \\
\text { tual Support } \\
\text { Program in } \\
\text { Dementia) }\end{array}$ & $\begin{array}{l}\text { Desenvolver } \\
\text { competências } \\
\text { de resolução } \\
\text { de problemas } \\
\text { familiares. }\end{array}$ & 80 Pacientes & $\begin{array}{l}\text { Estudo } \\
\text { clínico } \\
\text { random- } \\
\text { izado } \\
\text { controlado }\end{array}$ & $\begin{array}{l}\text { Family Caregiving Burden Inven- } \\
\text { tory; World Health Organization } \\
\text { Quality of Life Measure, Abbreviat- } \\
\text { ed version; Six-item Social Support } \\
\text { Questionnaire; MMS-E. }\end{array}$ & $\begin{array}{l}8 \text { Sessões } \\
\text { quinzenais de } \\
120 \text { minutos. }\end{array}$ & $\begin{array}{l}\text { Melhoria } \\
\text { significativa na } \\
\text { sobrecarga e na } \\
\text { qualidade de } \\
\text { vida; Atraso na } \\
\text { deterioração } \\
\text { física e cognitiva } \\
\text { dos pacientes } \\
\text { e melhoria no } \\
\text { suporte social. }\end{array}$ \\
\hline $\begin{array}{l}\text { Guerra, } \\
\text { Ferri, Fonseca, } \\
\text { Banerjee, e } \\
\text { Prince (2010) }\end{array}$ & $\begin{array}{l}\text { Peru } \\
\text { Helping car- } \\
\text { ers to care: } \\
\text { the } 10 / 66 \\
\text { dementia } \\
\text { research } \\
\text { group's }\end{array}$ & $\begin{array}{l}\text { Testar a } \\
\text { efetividade do } \\
\text { programa de } \\
\text { intervenção. }\end{array}$ & 58 Cuidadores & $\begin{array}{l}\text { Estudo } \\
\text { clínico } \\
\text { random- } \\
\text { izado } \\
\text { controlado }\end{array}$ & $\begin{array}{l}\text { Cuidador: Zarit Burden Interview, } \\
\text { psychological distress, quality of life. } \\
\text { Paciente: scales assessing behavioral } \\
\text { and psychological symptoms (NPI- } \\
\text { Q), quality of life (DEMQOL). }\end{array}$ & $\begin{array}{l}5 \text { Semanas } \\
\text { com sessões } \\
\text { semanais de } \\
30 \text { min. }\end{array}$ & $\begin{array}{l}\text { Diminuição da } \\
\text { sobrecarga; }\end{array}$ \\
\hline $\begin{array}{l}\text { Kurz, } \\
\text { Wagenpfeil, } \\
\text { Johannes, } \\
\text { Schneider- } \\
\text { Schelte, \& } \\
\text { Jansen (2010) }\end{array}$ & $\begin{array}{l}\text { Alemanha, } \\
\text { Suíça e } \\
\text { Áustria } \\
\text { Deutsche } \\
\text { Alzheimer } \\
\text { Gesellschaft - } \\
\text { DAG) }\end{array}$ & $\begin{array}{l}\text { Transmitir } \\
\text { informa- } \\
\text { ção sobre a } \\
\text { Doença de } \\
\text { Alzheimer }\end{array}$ & $\begin{array}{l}292 \text { Cuida- } \\
\text { dores }\end{array}$ & $\begin{array}{l}\text { Estudo } \\
\text { clínico } \\
\text { random- } \\
\text { izado } \\
\text { controlado }\end{array}$ & $\begin{array}{l}\text { Entrevista de aconselhamento não } \\
\text { estruturada; }\end{array}$ & $\begin{array}{l}7 \text { Sessões } \\
\text { quinzenais de } \\
90 \text { min cada }\end{array}$ & $\begin{array}{l}\text { Melhoria não } \\
\text { significativa na } \\
\text { qualidade de } \\
\text { vida psicológica } \\
\text { dos cuidadores; } \\
\text { Ausência de efei- } \\
\text { to na depressão } \\
\text { e no de interna- } \\
\text { mentos. }\end{array}$ \\
\hline
\end{tabular}

\section{DISCUSSÃO}

A maioria dos programas avaliados apresentam resultados positivos relativamente à diminuição da sobrecarga dos cuidadores de pessoas com demência após a intervenção psicoeducacional.

Nos estudos realizados por Ducharme, Lachance, Lévesque, Kergoat e Zarit (2012) referem que para promoção da saúde mental dos cuidadores é imperioso adequar as intervenções como a avaliação cognitiva do stress, uso de estratégias de resolução de problemas, gestão de stress e sintomatologia. Através do estudo realizado verificou-se uma melhoria pouco significativa nos indicadores de saúde do cuidador. Segundo Guerra, Ferri, Fonseca, Banerjee e Prince (2010) os resultados observados mostraram uma diminuição na sobrecarga, no entanto, não evidenciaram diferenças na qualidade de vida dos cuidadores e pacientes.

A Associação Alemã de Alzheimer (Deutsche Alzheimer Gesellschaft - DAG) baseou o seu programa em intervenções breves na vertente educativa e na técnica de resolução de problemas. Verificamos que no estudo realizado o efeito da intervenção é pequeno na qualidade de vida psicológica dos cuidadores não refletindo qualquer efeito na diminuição da sintomatologia depressiva dos cuidadores, assim como, a admissão em lares e o número de internamentos aumentou no grupo de intervenção. Verifica-se neste estudo uma melhoria do estado psicológico dos cuidadores não sendo possível atribuir fatores específicos, remetendo-nos para o suporte social fornecido. O programa Family Mutual Support Program in Dementia foi desenvolvido para 80 pacientes chineses residentes em Hong Kong e seus cuidadores, com especial atenção nos aspetos culturais e valores, os cuidadores mais experientes são os formadores dos novos cuidadores. Neste estudo verificou-se uma melhoria significativa na sobrecarga e na qualidade de vida dos cuidadores, atraso na deterioração física e cognitiva dos pacientes e melhoria no suporte social. O START - Strategies for Relatives, é um programa implementado no Reino Unido por psicólogos sem experiência clínica e individualmente, de forma a melhorar o encontro com os cuidadores, durante as sessões e diminuindo os custos do programa. 
Os resultados do programa START foram estatisticamente significativos, detetou-se diminuição do número de casos clínicos de depressão e diminuição dos níveis de ansiedade e, por conseguinte, melhoria na qualidade de vida dos cuidadores e diminuição dos comportamentos abusivos do cuidado. O programa psicoeducacional de apoio a cuidadores de doentes com demência - CUIDA foi realizado no âmbito da tese de doutoramento de Pereira (2015) verificou-se que a sobrecarga aumenta com o estadio da doença onde existe um agravamento da sintomatologia, como as alterações comportamentais e psicológicas e a perda de autonomia.

O programa psicoeducacional desenvolvido por Lopes e Cachioni (2012), no Brasil, de forma a avaliar o impacto deste programa para cuidadores familiares de pacientes com demência, relacionando com o bemestar subjetivo. Verificamos que após a aplicação do programa os cuidadores apresentaram satisfação de um modo geral com a vida e socialmente. Detetou-se que os rendimentos auferidos pelo agregado familiar estão interligados à satisfação geral com a vida, bem como, o sexo, género, parentesco, rendimentos, idade e tempo disponibilizado para o cuidar. Obteve-se um impacto positivo no bem-estar subjetivo, na satisfação geral com a vida, no envolvimento social e no equilíbrio entre afetos positivos e negativos. Segundo as variáveis estudadas verifica-se que em relação ao género, os cuidadores masculinos tiveram um aumento na satisfação geral com a vida, a nível social e dos afetos positivos com diminuição da sintomatologia depressiva. Por outro lado, as mulheres beneficiaram do aumento da satisfação com a capacidade mental e dos afetos positivos após a intervenção. Quanto à variável idade os cuidadores do sexo masculino foram os que mais beneficiaram, com idade inferior a 60 anos, os filhos e outros parentes (como irmãos, sobrinhos e netos), os cuidadores com menores rendimentos e os que cuidavam há mais de 12 meses. As diferenças entre os graus de parentesco de relação cuidador/idoso mostraram que os filhos aumentaram a satisfação geral com a vida, saúde, capacidade mental, envolvimento social e aumento dos afetos positivos, no entanto houve diminuição da satisfação com a capacidade física. Já os cuidadores cônjuges foram os que menos beneficiaram pois verificou-se alterações somente na satisfação a nível social. Os cuidadores com rendimentos baixos estão mais adaptados fazendo face aos problemas de uma forma mais eficaz verificandose melhoria na capacidade mental, envolvimento social e aumento dos afetos positivos, devido às informações fornecidas e ao suporte social que antes não possuíam.
Os cuidadores com mais recursos financeiros sentemse mais insatisfeitos, possivelmente, pelas expectativas que tinham para este momento da vida e não em exercer o papel de cuidador levando ao desgaste emocional. Quando avaliada a variável do tempo de cuidado verificou-se que os cuidadores mais jovens e que cuidavam há mais de 12 meses tiveram aumento da satisfação com a saúde e socialmente, diminuição da sintomatologia depressiva e aumento de afetos positivos, possivelmente, pelo facto de estarem já acomodados a esta condição de cuidar. Por outro lado, os cuidadores que cuidavam há menos tempo tiveram ligeiro aumento da satisfação pessoal.

No estudo "Cuidar em Casa", verifica-se que existe uma diminuição dos níveis de stress psicológico, após a implementação do programa, devendo ser abordado de forma multidisciplinar, de modo a adotar estratégias mais eficazes de coping, aquisição e desenvolvimento de competências.

\section{CONCLUSÕES}

A sobrecarga é um fator que influencia a qualidade de vida dos pacientes e dos seus cuidadores. Os resultados dos programas psicoeducacionais apontam para uma melhoria não só da sobrecarga, mas também para um aumento do bem-estar, aquisição de estratégias de resolução de problemas, aumento das competências para lidar nas atividades de vida diárias, diminuição de sentimentos de desesperança, fortalecimento na comunicação e colaboração, através de uma melhor perceção sobre o grau de comprometimento que a pessoa com demência apresenta.

Contudo, nos programas avaliados verifica-se que não existe uma padronização da intervenção, relativamente aos conteúdos programáticos, ao número de participantes e duração das sessões, o que dificulta a comparação dos resultados obtidos e a implementação desses resultados. A avaliação da eficácia dos programas é superficial, tornando-se necessário perceber a necessidade da manutenção dos ganhos obtidos no decorrer do tempo e os custos associados a estas intervenções

As limitações da revisão integrativa prendem-se com os critérios de inclusão estabelecidos, como a utilização de artigos e Português, Inglês e Espanhol e em acesso livre, o que pode ter limitado o acesso a outros trabalhos relevantes já realizados. Contudo, apesar dos programas de intervenção psicoeducativa, a nível internacional, serem alvo de estudo; a nível nacional, verificam-se ainda poucos estudos acerca dos programas de intervenção psicoeducativa, evidenciando a pertinência de se aprofundar esta problemática. 


\section{IMPLICAÇÕES PARA A PRÁTICA CLÍNICA}

De acordo com os resultados obtidos neste estudo verifica-se que os programas interdisciplinares de educação e suporte para cuidadores, proporcionam a redução dos níveis de sobrecarga do cuidador. Por conseguinte, é necessário a implementação e ampliação de intervenções efetivas, de forma a melhorar quer a qualidade de vida do cuidador, quer da pessoa com demência, e incentivar os profissionais para a realização destes programas, abordando também técnicas para gerir sintomas psicológicos e comportamentais.

\section{REFERÊNCIAS BIBLIOGRÁFICAS}

American Psychiatric Association. (2013). Diagnostic and statistical manual of mental disorders (5th ed.). Washington DC: American Psychiatric Press.

Aakhus, E., Engedal, K., Aspelund, T., \& Selbæk, G. (2009). Single session educational programme for caregivers of psychogeriatric in patients: results from a randomized controlled pilot study. International Journal of Geriatric Psychiatry, 24, 269-274.

Alves, S., Brandão, D., Teixeira, L., Azevedo, M. J., Duarte, M., Ribeiro, O., e Paúl, C. (2015). Intervenções psicoeducativas e distress psicológico em cuidadores informais: Análise comparativa de dois projetos comunitários. Revista Eletrónica de Psicologia, Educação e Saúde, 5(1), 94-112.

Cardoso, M. J. S. P. O. (2011). Promover o bem-estar do familiar cuidador: Programa de intervenção estruturado (Doutoramento). Porto: Universidade Católica Portuguesa.

Chien, W. T., \& Lee, Y. M. (2008). A disease management program for families of persons in Hong Kong with dementia. Psychiatric Services, 59(4), 433-436.

Chien, L. Y., Chu, H., Guo, J. L., Liao, Y. M., Chang, L. I., \& Chen C. H. (2011). Caregiver support groups in patients with dementia: A meta-analysis. International Journal of Geriatric Psychiatry, 26(10), 1089-1098.

Cruz, J., Barbosa, A., Figueiredo, D., Marques, A., e Sousa, L. (2015). Cuidar com sentido(s): Guia para cuidadores de pessoas com demência. Aveiro: Universidade de Aveiro.
Ducharme, F., Lachance, L., Lévesque, L., Kergoat, M. J., \& Zarit, S. H. (2012). Persistent and delayed effects of a psycho-educational program for family caregivers at disclosure of dementia diagnosis in a relative: A sixmonth follow-up study. Healthy Aging Research, 1(2), $1-11$.

Gallagher-Thompson, D., Coon, D. W., Solano, N., Ambler, C., Rabinowitz, Y., \& Thompson L. W. (2003). Change in indices of distress among Latino and Anglo female caregivers of elderly relatives with dementia: Site-specific results from the REACH national collaborative study. Gerontologist, 43(4), 580-591.

Guerra, M., Ferri, C. P., Fonseca, M., Banerjee, S., \& Prince M. (2010). Helping carers to care: The 10/66 dementia research group's randomized control trial of a caregiver intervention in Peru. Revista Brasileira de Psiquiatria, 33(1), 47-54.

HelpAge International. (2017). Ageing in the 21st century: A celebration and a challenge. London: HelpAge International.

Kurz, A., Wagenpfeil, S., Johannes, H., ScheinederSchelte, H., \& Jansen, S. (2010). Evaluation of a brief educational program for dementia carers: The AENEAS Study. International Journal of Geriatric Psychiatry, 25, 861-869.

Lazarus, R. S., \& Folkman, S. (1984). Stress, appraisal and coping. New York: Springer Publishing.

Livingston, G., Barber, J., Rapaport, P., Knapp, M., Griffin, M., King, D., \& Cooper, C. (2013). Clinical effectiveness of a manual based coping strategy programme (START, STrAtegies for Relatives) in promoting the mental health of carers of family members with dementia: Pragmatic randomised controlled trial. BMJ, 2013, 347. doi: 10.1136/bmj.f6276

Lopes, L., e Cachioni, M. (2012). Intervenções psicoeducacionais para cuidadores de idosos com demência: Uma revisão sistemática. Jornal Brasileiro de Psiquiatria, 61(4), 252-261.

Marques, S. L. (2007). Cuidadores informais de doentes com acidente vascular cerebral. Coimbra: Formasau. 
Mendes, K., Silveira, R., \& Galvão, C. (2008). Integrative review: A research method to incorporate evidence in health care and nursing. Texto \& Contexto Enfermagem, 17(4), 758-764.

Pereira, F. (2015). Necessidades dos cuidadores de doentes com demência (Tese de Doutoramento). Lisboa: Universidade Autónoma de Lisboa.
Sequeira, C. (2010). Cuidar de idosos com dependência física e mental. Lisboa: Lidel.

Sousa, L., Mendes, A., e Relvas, A. P. (2007). Enfrentar a velhice e a doença crónica. Lisboa: Climepsi.

Wang, L., \& Chien, W. (2011). Randomised controlled trial of a family-led mutual support programme for people with dementia. Journal of Clinical Nursing, 20, 2362-2366. 